\title{
Two-photon absorption study of GaN
}

\author{
Chi-Kuang Sun, ${ }^{\text {a) }}$ Jian-Chin Liang, and Jiun-Cheng Wang \\ Department of Electrical Engineering and Graduate Institute of Electro-Optical Engineering, \\ National Taiwan University, Taipei 10617, Taiwan, Republic of China \\ Fu-Jen Kao \\ Department of Physics, National Sun Yat-Sen University, Kaoshiung 80424, Taiwan, Republic of China
}

Stacia Keller, Michael P. Mack, Umesh Mishra, and Steven P. DenBaars

Department of Electrical and Computer Engineering and Materials Department, University of California, Santa Barbara, California 93106

(Received 21 July 1999; accepted for publication 24 November 1999)

Two-photon absorption coefficients of GaN for below band gap ultraviolet wavelength and midgap infrared wavelength were measured by using femtosecond pulsewidth autocorrelation and Z-scan techniques. Large two-photon absorption coefficients were obtained. Taking advantage of the large two-photon absorption, we have demonstrated two-photon confocal imaging of a GaN thin film. Direct correlation was found between the yellow luminescence and suppression of bandedge luminescence. (C) 2000 American Institute of Physics. [S0003-6951(00)04404-1]

$\mathrm{GaN}$ and its related materials have attracted great interest for their applications in light emitters in the green to ultraviolet (UV) wavelength. ${ }^{1}$ Recently there has been a growing attention on the studies of the two-photon absorption (TPA) process in GaN, which will lead to optical power limitation and optical damages in nitride based optoelectronics. Taking advantage of the TPA process in GaN thin films and photodetectors, autocorrelation and cross-correlation of visible and infrared (IR) femtosecond pulses have been demonstrated by several research groups. ${ }^{2-5}$ TPA induced photoluminescence (PL) of GaN was also previously studied ${ }^{6}$ using tunable picosecond pulses. A large TPA coefficient $\beta$ of $17.5 \mathrm{~cm} / \mathrm{G} \mathrm{W}$ at $600 \mathrm{~nm}$ wavelength was reported. ${ }^{2}$ A value of $\sim 1.5 \mathrm{~cm} / \mathrm{G} \mathrm{W}$ was also obtained for $\beta$ at photon energies above $E_{\text {gap } / 2}$ in the IR wavelength region. ${ }^{7}$

In this letter, we report our study of the TPA of $\mathrm{GaN}$ in below band gap UV wavelength region using TPA-type autocorrelation techniques. The study of TPA process for below band gap UV-blue wavelength is important due to the fact that most nitride based optoelectronics are operated in this wavelength regime instead of in red or IR wavelength. On the other hand, optical autocorrelation of ultrashort pulses using TPA provides a convenient, sensitive, and inexpensive alternative to standard techniques using nonlinear crystals. ${ }^{8-10}$ UV TPA autocorrelation is especially important due to the difficulty of finding a suitable second-harmonic generation crystal in this wavelength regime. In order to avoid possible systematic errors by the two-photon autocorrelation technique, we have also performed single-beam Z-scan measurements for below band gap UV wavelengths.

The GaN sample was grown by atmospheric-pressure metalorganic chemical-vapor deposition on a doubleside polished $c$-plane sapphire substrate. After annealing the substrate and deposition of a nucleation layer, unintentionally doped GaN layer of $5 \mu \mathrm{m}$ thickness was grown. The crystal structure is wurzite. A room-temperature transmission spec-

a)Electronic mail: sun@cc.ee.ntu.edu.tw trum indicated that the band gap of the sample was located $\sim 365 \mathrm{~nm}$. The TPA study was performed by demonstrating UV TPA autocorrelation using a standard transmission-type pump-probe technique. The laser output from a femtosecond mode-locked Ti:sapphire laser was frequency doubled in a $500-\mu \mathrm{m}$-thick betabarium-borate crystal to reach UV wavelength. The frequency-doubled UV pulses were tunable between 350 and $400 \mathrm{~nm}$ with a repetition rate of $82 \mathrm{MHz}$. The UV pulses were split as pump and probe pulses by a beamsplitter. These two beams were then focused onto the same point on the GaN sample by using an objective. The pump beam was chopped and the detected probe signal was measured as a function of the temporal delay between the pump and the probe by a lock-in amplifier. Complimentary transient reflection measurements were also performed in order to remove the transient contributions due to surface reflection.

Figure 1(a) shows the measured transient response using UV femtosecond pulses centered at a wavelength of $390 \mathrm{~nm}$ (open circles). Around zero time delay, a large transmission decrease was observed. This was induced by the TPA process. The differential transmission change of probe beam decreased linearly with the pump excitation power, indicating a TPA process consisted of one pump photon and one probe photon. The shape of the TPA-induced differential transmission decrease will mimic the pulse autocorrelation function. However, after the TPA decrease, the measured transient response did not return to zero but was followed by a small step-like positive transmission increase within our measured time delay $(\sim 1 \mathrm{ps})$. This transmission increase is attributed to the absorption saturation of the bandtail states. This bandtail state absorption might be the origin of the nonideal interferometric autocorrelation pulse shape (a 6:1 ratio instead of an 8:1 ratio) obtained from a GaN photodetector using $410 \mathrm{~nm}$ pulses. ${ }^{4}$ In order to remove the contribution from the bandtail state saturation, we have performed a convolution fit. The fitting considers two components: a Gaussian pulse shape (negative, representing the TPA induced pulse- 

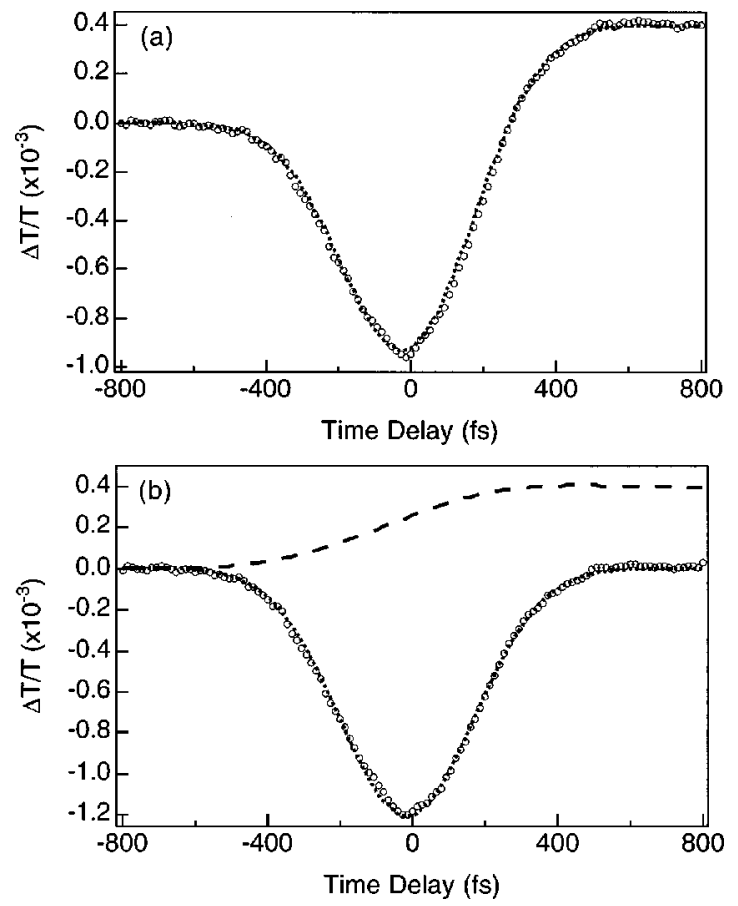

FIG. 1. (a) Transient transmission response (open circles) with $390 \mathrm{~nm}$ UV pulses. The dotted line is a convolution fit. (b) The modified data (open circles) after removing the contribution from the convoluted step response (dashed line). The dotted line is a Gaussian fit.

width autocorrelation) and its convolution with a step function (positive, representing the absorption saturation contribution). The dotted line on top of the experimental data is a convolution fit. The dotted line and the dashed lines in Fig. 1(b) are the corresponding contributions from the negative Gaussian pulse shape and its positive convolution component with the step function. The open circles in Fig. 1(b) are the corrected TPA autocorrelation trace after removing the step function contribution from the experimental data. An excellent fit with a Gaussian pulse shape was obtained. An autocorrelation full width at half maximum (FWHM) of $430 \mathrm{fs}$ was obtained for this specific trace. A FWHM pulsewidth of 300 fs can thus be derived. The focal spot diameter of the UV beam, at which the electric field amplitude of the Gaussian beam decreases to $1 / \mathrm{e}$ of its peak value, was measured to be $15.7 \mu \mathrm{m}$ determined by transmission through a $10 \mu \mathrm{m}$ pinhole. The pump pulse energy inside the sample was 200 pJ, corrected after the surface Fresnel reflection. Considering the Gaussian spatial and temporal intensity profiles, a TPA coefficient of $12 \pm 6 \mathrm{~cm} / \mathrm{G} \mathrm{W}$ can thus be derived from the peak transmission decrease of $0.12 \%$ assuming a complete pump probe overlap.

Similar measurements were also performed at wavelength between 400 and $377 \mathrm{~nm}$. Values of $\beta$ between $16 \pm 7$ and $12 \pm 6 \mathrm{~cm} / \mathrm{GW}$ were obtained. For wavelength shorter than $375 \mathrm{~nm}$, the transient response was found to be dominated by absorption saturation of bandtail states, which will result in greater fitting errors. In order to confirm these measured results, we have also performed Z-scan measurements on the GaN sample. ${ }^{11}$ TPA coefficients of $17 \pm 7$ and $14 \pm 6$ $\mathrm{cm} / \mathrm{G} \mathrm{W}$ can be derived from Z-scan traces at wavelengths of 387 and $391 \mathrm{~nm}$, respectively. These measured TPA coefficients of GaN at UV wavelengths are much larger than the reported UV TPA coefficients of diamond $(0.75 \mathrm{~cm} / \mathrm{G} \mathrm{W}$ at

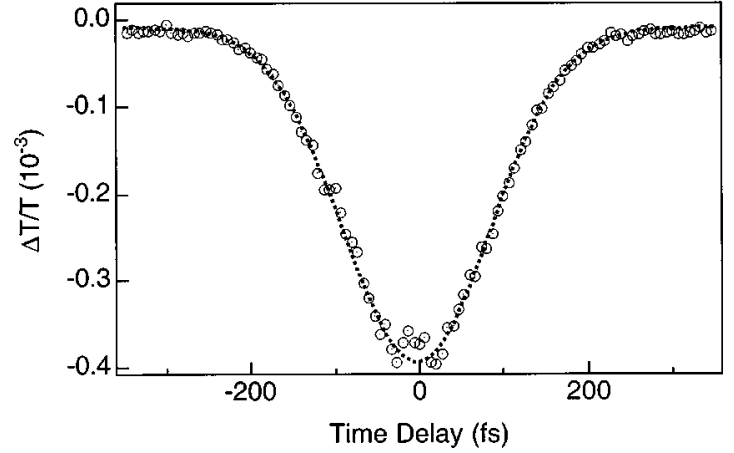

FIG. 2. $720 \mathrm{~nm}$ IR pulse autocorrelation measured by TPA-induced transmission decrease in a GaN thin film.

$310 \mathrm{~nm},{ }^{12} 2.3 \mathrm{~cm} / \mathrm{GW}$ at $\left.282 \mathrm{~nm}\right)^{13}$ and fused silica $(0.045$ $\mathrm{cm} / \mathrm{GW}$ at $267 \mathrm{~nm}),{ }^{10}$ which have both been proposed as candidate TPA materials for UV pulse autocorrelation. The measured and thus obtained large TPA coefficients of GaN indicate great potential of nitride-based semiconductors as nonlinear crystals for visible-UV photonic applications. Even though the applicable wavelength is limited by the band gap energy of GaN ( $365 \mathrm{~nm}$ ), it can be extended further into UV wavelength by adding aluminum composition into $\mathrm{GaN}$.

Experiments were also performed at above midgap IR wavelength. With a band gap energy around $365 \mathrm{~nm}$, TPAinduced band-to-band transition in GaN will allow photons with wavelength up to $730 \mathrm{~nm}$. At above midgap IR wavelength, no defect transition is expected and a symmetric autocorrelation function should thus be obtained. Figure 2 shows a measured transient transmission trace on a $2.5-\mu \mathrm{m}-$ thick GaN thin film using $720 \mathrm{~nm}$ fs pulses. A symmetric TPA-induced response was obtained, corresponding to the pulse autocorrelation function. The dotted line in Fig. 2 shows a Gaussian fit with a FWHM of 200 fs. A pulsewidth of $140 \mathrm{fs}$ can thus be derived. The pump pulse energy inside the sample was $236 \mathrm{pJ}$ with a focal beam diameter of 16.3 $\mu \mathrm{m}$. A maximum probe transmission decrease of $0.04 \%$ will thus correspond to a $\beta$ of $3 \pm 1.5 \mathrm{~cm} / \mathrm{G} \mathrm{W}$ at $720 \mathrm{~nm}$ wavelength. At $690 \mathrm{~nm}$ wavelength, a $\beta$ of $7 \pm 3 \mathrm{~cm} / \mathrm{GW}$ was also obtained in a similar measurement procedure.

Figure 3 summarizes the measured results. Open circles represent the values obtained from TPA autocorrelation measurements, while open triangles represent the values from Z-scan measurements. ${ }^{11}$ Previously reported value ${ }^{2}$ at 600 $\mathrm{nm}$ is also displayed as a solid diamond for comparison. According to the study of Sheik-Bahae and his co-workers, TPA coefficient $\beta$ of a direct band gap semiconductor at a photon energy $h \nu$ can be described by ${ }^{14}$

$$
\beta(h \nu)=K \sqrt{\frac{E_{p}}{n_{o}^{2} E_{g}^{3}}} F_{2}\left(\frac{h \nu}{E_{p}}\right),
$$

with $F_{2}(x)=(2 x-1)^{1.5} /(2 x)^{5}$. $\mathrm{K}$ is 1940 while $\beta$ is in unit of $\mathrm{cm} / \mathrm{GW} . E_{p}$ is $\sim 21 \mathrm{eV} . E_{g}$ is the band gap energy of $\mathrm{GaN} . n_{o}$ is the refractive index of GaN. ${ }^{15}$ We plot 12 times of Eq. (1) as a dotted line in Fig. 3 for reference. Our measured results agree with the frequency dependence described by Eq. (1) but are larger by a constant, which is commonly observed in wide-band gap dielectrics. ${ }^{14}$ One of the possible reasons for the observed large constant is the negligence of 


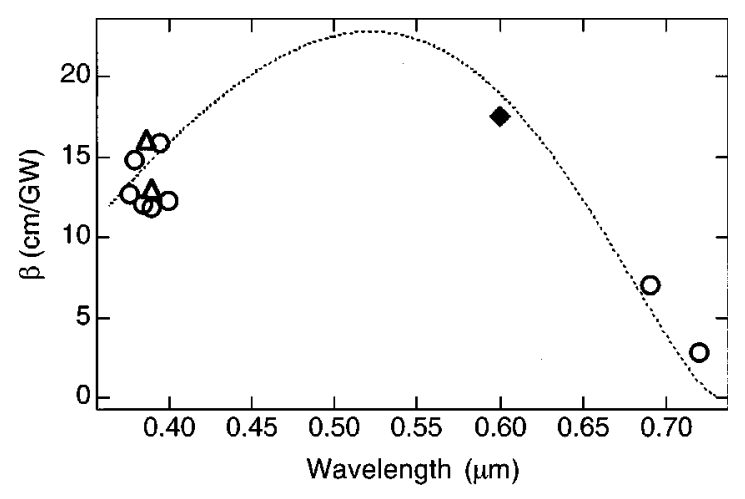

FIG. 3. TPA coefficient $\beta$ vs wavelength obtained by autocorrelation measurements (open circles) and $\mathrm{Z}$ scans (open triangles). Previously reported data from Ref. 2 is displayed as a solid diamond for reference. The dotted line is 12 times of Eq. (1).

exciton effects in Eq. (1), while exciton effects are significant in GaN and are found to enhance TPA coefficient. ${ }^{16}$

Taking advantage of the large TPA coefficient of GaN at above midgap IR wavelength, two-photon confocal scanning microscopy ${ }^{17}$ on GaN can be easily demonstrated using femtosecond IR pulses. The use of IR wavelength leads to a deeper penetration depth in most materials, providing an opportunity to image thicker samples. For GaN studies, the use of IR excitation wavelength can also avoid most expensive UV optics. Figures 4(a) and 4(b) show the measured twophoton scanning PL images of a GaN Hall-measurement sample. Femtosecond pulses at wavelength of $720 \mathrm{~nm}$ were passed through XY galvano mirrors to perform twodimensional scanning. The dithered laser beam was then directed into an inverted optical microscope (Olympus BX50). A $10 \times$ objective was utilized to focus the laser beam onto the $\mathrm{GaN}$ layer. The valence band carriers were photoexcited into the conduction band by strong TPA processes and the subsequent PL was collected by the same microscope objective, separated from the input laser beam by a dichroic beamsplitter, and directed into a photomultiplier (PMT) tube. Due to the large wavelength difference between the excitation pulse and PL light, the PL photons can be easily separated from the pump laser beam. Combining with strong TPA in $\mathrm{GaN}$, large signal-to-noise ratio can thus be easily achieved. Figure 4(a) shows an image taken with a $365 \mathrm{~nm}$ interference filter with $10 \mathrm{~nm}$ bandwidth in front of the PMT tube, corresponding to the $\mathrm{GaN}$ band gap wavelength at room temperature. Figure 4(b) shows an image taken with a 550-650 $\mathrm{nm}$ bandpass filter, corresponding to the wavelength of the defect yellow luminescence. Figure 4(c) shows an image taken with the transmitted $720 \mathrm{~nm}$ beam through the sample. The image was taken from the edge of the Hall-measurement sample with two oval defects. Figure 4(a) indicates excellent lateral uniformity in the measured sample except on the areas around oval defects. It is interesting to notice that the area emitting weak edge PL corresponds to the area emitting strong yellow PL. The yellow PL of GaN has been thought to compete with the edge PL and is studied intensively recently. ${ }^{18}$ Our demonstration indicates that two-photon confocal microscopy of $\mathrm{GaN}$ cannot only exhibits superior image quality but also reveals interesting details that are not readily available though other contrast mechanisms.

In summary, we have studied TPA of GaN by demon-
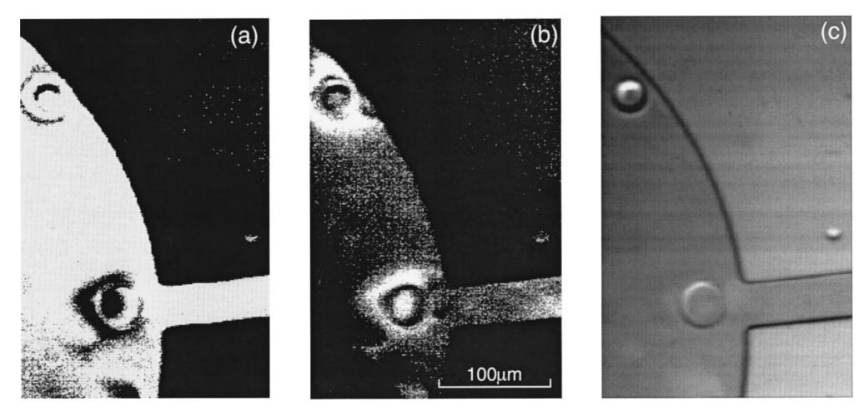

FIG. 4. Two-photon confocal PL images of a GaN Hall-measurement sample. The wavelength of the excitation pulses is $720 \mathrm{~nm}$. (a) Detected PL image with a $365 \mathrm{~nm}$ filter. (b) Detected PL image with a 550-650 nm bandpass filter. (c) Detected transmission image by using the $720 \mathrm{~nm}$ pulses.

strating UV two-photon autocorrelation. Large TPA coefficient $\beta$ at below band gap UV wavelengths was observed, indicating $\mathrm{GaN}$ and it related materials as excellent candidates for UV nonlinear applications. Large $\beta$ at abovemidgap IR wavelengths was also observed. Taking advantages of this large $\beta$ at above-midgap IR wavelengths, we have demonstrated two-photon confocal microscopy on a $\mathrm{GaN}$ Hall-measurement sample using IR femtosecond pulses. Direct correlation was found between yellow luminescence and suppression of bandedge PL.

This project is sponsored by National Science Council of Taiwan, Republic of China under Grant No. NSC 88-2112M-002-003. The work at UCSB is supported by a grant from NSF QUEST Science and Technology Center.

${ }^{1}$ S. Nakamura, M. Senoh, N. Iwasa, T. Yamada, T. Matsushita, H. Kiyoku, and Y. Sugimoto, Jpn. J. Appl. Phys., Part 2 35, L74 (1996).

${ }^{2}$ S. Petit, D. Guennani, P. Gilliot, C. Hirlimann, B. Hönerlage, O. Briot, and R. L. Aulombard, Mater. Sci. Eng., B 43, 196 (1997).

${ }^{3}$ A. Wada, W. Ho, and M. A. Khan, Thin Solid Films 306, 137 (1997).

${ }^{4}$ A. M. Streltsov, A. L. Gaeta, P. Kung, D. Walker, and M. Razeghi, in Technical Digest of Conference on Laser and Electro-optics, Baltimore, MD, paper CFG6 (1999).

${ }^{5}$ C.-K. Sun, Y.-L. Huang, J.-C. Wang, S. Keller, M. Mack, U. K. Mishra, and S. P. DenBaars, in Technical Digest of Conference on Laser and Electro-optics, Baltimore, MD, paper CTuI4 (1999).

${ }^{6}$ D. Kim, I. H. Libon, C. Voelkmann, Y. R. Shen, and V. Petrva-Koch, Phys. Rev. B 55, 4907 (1997).

${ }^{7}$ J. Miragliotta and D. K. Wickenden, Appl. Phys. Lett. 69, 2095 (1996).

${ }^{8}$ D. T. Reid, M. Padgett, C. McGrowan, W. E. Sleat, and W. Sibbett, Opt. Lett. 22, 233 (1997)

${ }^{9}$ W. Rudolph, M. Sheik-Bahae, A. Bernstein, and L. F. Lester, Opt. Lett. 22, 313 (1997).

${ }^{10}$ A. M. Streltsov, J. K. Ranka, and A. L. Gaeta, Opt. Lett. 23, 798 (1998).

${ }^{11}$ Y.-L. Huang, C.-K. Sun, J.-C. Liang, S. Keller, M. P. Mack, U. K. Mishra, and S. P. DenBaars, Appl. Phys. Lett. 75, 3524 (1999).

${ }^{12}$ J. I. Dadap, G. B. Focht, D. H. Reitze, and M. C. Downer, Opt. Lett. 16, 499 (1991).

${ }^{13}$ A. Reuther, A. Laubereau, and D. N. Nikogosyan, Opt. Commun. 141, 180 (1997).

${ }^{14}$ M. Sheik-Bahae, D. C. Hutchings, D. J. Hagan, and E. W. Van Stryland, IEEE J. Quantum Electron. 27, 1296 (1991).

${ }^{15}$ G. Yu, G. Wang, H. Ishikawa, M. Umeno, T. Soga, T. Egawa, J. Watanabe, and T. Jimbo, Appl. Phys. Lett. 70, 3209 (1997).

${ }^{16}$ C. C. Lee and Y. H. Fan, Phys. Rev. B 9, 3502 (1974).

${ }^{17}$ W. Denk, J. H. Strickler, and W. W. Webb, Science 248, 73 (1990).

${ }^{18}$ M. Godlewski, E. M. Goldys, M. R. Phillips, R. Langer, and A. Barski, Appl. Phys. Lett. 73, 3686 (1998). 